\title{
Allergic diseases of the skin and drug allergies - 2030. Validation of cephalosporin skin test for prediciting immediate hypersensitivity: interim analysis
}

Sun-Young Yoon ${ }^{1 *}$, So Young Park', Sujeong Kim', Hyouk-Soo Kwon, Yun-Jeong Bae², Jaehyung Roh ${ }^{3}$, You Sook Cho', Tae-Bum Kim', Hee-Bom Moon ${ }^{4}$

From 2nd WAO International Scientific Conference (WISC 2012)

Hyderabad, India. 6-9 December 2012

\section{Background}

Cephalosporin is one of the most commonly used beta-lactam antibiotics globally, and also a major offending agent of drug hypersensitivity along with penicillin. Cephalosporin skin test has been widely used in most hospitals in Korea. However, the validity of this test for prediction of immediate hypersensitivity has still not been well studied. Therefore, we conducted this study to determine the predictive validity of cephalosporin skin test prior to administration.

\section{Methods}

We prospectively conducted intradermal skin test with selected $1^{\text {st }}, 2^{\text {nd }}$, and 3 rd generation cephalosporins: ceftazol, cefotetan or cefamandol, ceftriaxone or cefotaxime or flomoxef, respectively. After skin test, one of the tested cephalosporins was intravenously administered for preoperative prevention of infection under careful observation, regardless of the skin test results.

\section{Results}

We recruited 1,125 patients who needed the use of preoperative cephalosporins. Eighty five patients (7.5\%) showed positive skin test to at least one cephalosporin. However, none of these patients showed immediate hypersensitivity reactions. Two patients who showed generalized urticaria and itching sense had negative skin test. The test showed sensitivity of $0 \%$, specificity of $92.4 \%$, negative predictive value of $99.8 \%$, and positive predictive value $0 \%$.

\section{Conclusions}

Routine skin test of cephalosporin prior to its administration is not valid for predicting immediate hypersensitivity with low sensitivity and positive predictive value. This study is currently ongoing for enrollment of larger study group.

\section{Author details}

'Division of Allergy and Clinical Immunology, Department of Internal Medicine, Asan Medical Center, University of Ulsan College of Medicine, Seoul, South Korea. ${ }^{2}$ Health Medicine, Health Screening and Promotion Center, Asan Medical Center, Seoul, South Korea. ${ }^{3}$ Bonghwa Hospital, Gyungsangbukdo, South Korea. ${ }^{4}$ Asian Medical Center, College of Medicine Ulsan University, South Korea.

Published: 23 April 2013

doi:10.1186/1939-4551-6-S1-P116

Cite this article as: Yoon et al:: Allergic diseases of the skin and drug allergies - 2030. Validation of cephalosporin skin test for prediciting immediate hypersensitivity: interim analysis. World Allergy Organization Journal 2013 6(Suppl 1):P116 